\title{
Pain Severity and Interference in Different Parkinson's Disease Cognitive Phenotypes
}

This article was published in the following Dove Press journal:

Journal of Pain Research

\author{
Yenisel Cruz-Almeida (D) \\ Samuel J Crowley ${ }^{2}$ \\ Jared Tanner ${ }^{2}$ \\ Catherine C Price ${ }^{2,3}$ \\ 'Pain Research \& Intervention Center of \\ Excellence, University of Florida, \\ Gainesville, FL, USA; ${ }^{2}$ Department of \\ Clinical and Health Psychology, University \\ of Florida, Gainesville, FL, USA; ${ }^{3}$ Norman \\ Fixel Institute for Neurological Diseases, \\ University of Florida, Gainesville, FL, USA
}

Correspondence: Catherine C Price

ABPP/Cn

$\mathrm{Tel}+$ I 352-273-5929

Email cep23@phhp.ufl.edu

\begin{abstract}
Introduction: Chronic pain is prevalent in idiopathic Parkinson's disease (PD) with many individuals also experiencing cognitive deficits negatively impacting everyday life.

Methods: In this study, we examine differences in pain severity and interference between 113 nondemented individuals with idiopathic PD who were statistically classified as having low executive function $(n=24)$, low memory function $(n=35)$, no cognitive deficits $(n=54)$. The individuals with PD were also compared to matched non-PD controls ( $n=64)$.

Results: PD participants with low executive function reported significantly higher pain interference $(p<0.05)$, despite reporting similar pain severity levels compared to other phenotypes. These differences remained statistically significant, even after accounting for important confounders such as anxiety and depression $(\mathrm{p}<0.05)$.
\end{abstract}

Discussion: Pain interference in those with lower executive function may represent a target for psychosocial interventions for individuals with pain and PD.

Keywords: Parkinson's disease, pain, cognitive function, profiles, phenotypes

\section{Introduction}

Idiopathic Parkinson's Disease (PD) affects at least 1.5 million people in the United States leading to disabling motor and non-motor impairments. Cognitive deficits are common in PD with patients experiencing problems across several domains. ${ }^{1-5}$ Chronic pain is also commonly reported by this population compared to agematched non-PD peers ${ }^{6-9}$ with pain being a significant source of disability that often precedes motor symptoms. ${ }^{10}$

Pain is a multidimensional experience made up of interacting sensory, motor, cognitive and emotional components. In regards to cognition, chronic pain sufferers often present with executive function deficits (see ${ }^{11}$ for a meta-analysis) and acute experimental pain reduces executive function abilities in healthy, pain-free individuals. $^{12,13}$ Pain inhibition and modulation is associated with prefrontal cortical regions, ${ }^{14}$ while executive dysfunction in PD is caused in part by dysfunction in cortico-striatal loops involving frontal regions. ${ }^{15}$ These similarities suggest that individuals with PD and executive difficulties may also experience more interference in their daily activities due to pain because of decreased function in prefrontal brain regions needed for pain inhibition.

The aim of this brief report was to compare self-reported pain severity and interference among individuals with PD shown in separate studies to have reduced executive or episodic memory function relative to cognitively healthy individuals with PD and non-PD peers. ${ }^{16,17}$ We compared pain severity and interference across 
three statistically derived clusters of PD cognitive phenotypes (PD Executive, PD Memory, and PD Cognitively Well). Based on known shared neural substrates of pain modulation and PD pathophysiology, we hypothesized individuals with PD and low performance in executive function tasks would experience significantly greater pain severity and pain interference compared to other patterns of cognitive functioning after controlling for relevant PD and pain-related variables.

\section{Methods}

\section{Participants}

This study was part of a federally funded investigation focused on cognitive function and $\mathrm{PD}$, in cooperation with the University of Florida's Center for Movement Disorder and Neurorestoration, with approval of the University of Florida's Institutional Review Board, and in compliance with the Declaration of Helsinki. Participants with PD were recruited through movement disorder clinic referrals and advertisements to local MDC support groups affiliated with the movement disorder clinic. Idiopathic PD was confirmed by a fellowship-level movement disorder specialist, using UK Parkinson's Disease Society Brain Bank Clinical Diagnostic Criteria. ${ }^{18}$ Individuals with early, mild to moderate PD with a Hoehn and Yahr scale ${ }^{19}$ score between 1 and 3 were included. The predominant motor PD phenotype; ie, tremor dominant vs postural instability-gait difficulty was assessed clinically based on the first presenting symptom or predominant symptomatology on initial and subsequent exams (and also of primary concern to the patient). Non-PD participants were recruited through 1) mailings to demographically similar individuals in two counties, 2) community fliers, and 3) free community memory screenings. Exclusion criteria included other neurodegenerative disorders, significant disease that could limit lifespan, major psychiatric disorder, or dementia determined from structured telephone interview and medical record review. Depression and apathy were not exclusion criteria due to high prevalence in PD. ${ }^{20,21}$ Participants in the current study provided informed consent and are a subset of participants also reported in a separate manuscript investigating PD cognitive phenotypes and brain structure. ${ }^{16}$

\section{Clinical Measures}

Measures were administered using standardized and validated methods as previously reported by our group and others. ${ }^{5,16}$ While on-medication, participants completed neuropsychological testing, as well as measures of 1) PD symptom severity (ie, Unified Parkinson's Disease Rating Scale (UPDRS ${ }^{22}$ ); 2) pain severity and interference (ie, the Brief Pain Inventory (BPI ${ }^{23}$ ); 3 ) disease comorbidity (ie, Charlson Comorbidity Scale ${ }^{24}$ ); and 4) depression (ie, the Geriatric Depression Scale; $\operatorname{GDS}^{25}$ ). Medications were reverted to a common metric and compared for dopaminergic (Levodopa Equivalency Dose; LED $^{26}$ ), and anticholinergic levels. ${ }^{27}$ Raters blinded to diagnosis double scored and double entered all data.

\section{Cognitive Measures and Cognitive Phenotypes}

The methods for deriving the cognitive phenotypes is described elsewhere. ${ }^{16}$ Cognitive phenotypes were derived from executive function and episodic memory measures including WAIS-III Letter-Number Sequencing and Digit Symbol tests, ${ }^{28}$ Stroop Color-Word Test color-word trial, ${ }^{29}$ Trail Making Test Part B ${ }^{30}$ Hopkins Verbal Learning Test, Revised delay and recognition discrimination measures, ${ }^{31}$ and WMS-III Logical Memory delay recall. ${ }^{32}$ The cluster analysis yielded three distinct cognitive phenotypes: 1) participants with the lowest performance across processing speed and executive tasks (PD Executive); 2) participants with the lowest performance in learning and memory tasks (PD Memory); and 3) participants with relatively normal performance across all measures (Cognitively Well). The final cluster solution underwent internal and external validation procedures. ${ }^{16,17}$

\section{Statistical Analyses}

The empirically derived clusters were reconfirmed relative to a previous report ${ }^{16}$ and compared across demographic and health-related measures using a one-way analysis of variance (ANOVA) for normally distributed, KruskalWallis ANOVAs for non-normally distributed continuous variables, and chi-square tests for nominal variables. Demographic and disease-related characteristics significantly different across clusters at a $\mathrm{p}<0.100$ were included as covariates in subsequent analyses. First, an ANCOVA compared pain severity between cognitive phenotypes and non-PD peers with age, sex, and years of education as covariates. PD phenotypes were also considered for differences in disease duration and motor severity. A second ANCOVA compared pain interference between cognitive phenotypes and non-PD peers, but with pain severity added as a covariate. Where the omnibus analysis was significant, 
post-hoc analyses compared the groups with a Bonferroni correction for multiple comparisons. Finally, these analyses were repeated with GDS as an additional covariate due to the established association between depression and both chronic pain ${ }^{33}$ and executive function. ${ }^{34} \mathrm{~A} p<0.05$ was considered statistically significant.

\section{Results}

\section{Cognitive Phenotypes}

The final sample included 113 individuals with PD and 60 non-PD peers; of the original recruitment sample, eight participants (3 PD, 5 non-PD) did not complete the BPI and thus were not included here. The three cognitive phenotypes consisted of 1) participants with the lowest performance across processing speed and executive tasks (PD Executive, $\mathrm{N}=24$ ); 2) participants with the lowest performance in episodic memory tasks (PD Memory, $\mathrm{N}=35$ ); and 3) participants with normal performance across all measures (PD Cognitively Well, N=54). Neuropsychology scores are provided (Supplementary Table 1). Groups differed significantly in years of education (Executive $<$ NonPD), disease duration (Memory $<$ Executive), UPDRS Part II (Cognitively Well < Executive), depressive symptom severity (Executive $<$ Non-PD), and anticholinergic burden (Non-PD $<$ Cognitively Well; Supplementary Table 2).

\section{Pain Differences - Cognitive Phenotypes}

After controlling for age, sex, disease duration, and education, there were no significant cluster differences in the BPI pain severity subscale with participants across all clusters reporting mild pain ratings. However, clusters differed significantly in the BPI pain interference subscale after controlling for these covariates. Post-hoc tests with Bonferroni correction showed that the PD Executive cluster reported significantly higher pain interference than Cognitively Well phenotype $(\mathrm{p}=0.01)$ and non-PD peers $(\mathrm{p}<0.01$, Supplementary Tables 3 and $\underline{4}$, Figure 1). Findings did not change significantly after controlling for GDS score (Supplementary Table 5).

\section{Discussion}

Individuals with PD who showed more difficulty on working memory and inhibitory function tests reported higher levels of pain interference than individuals with PD and no cognitive difficulties, as well as higher interference than non-PD peers. Yet, each PD group reported similar pain severity even after accounting for important PD and pain-

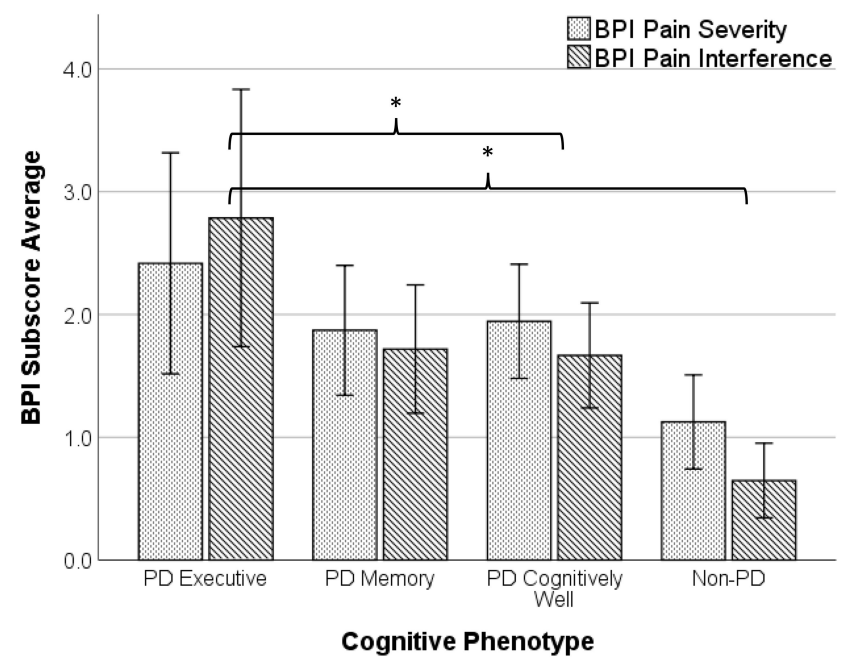

Figure I Brief Pain Inventory pain severity and pain interference across the cognitive phenotypes. *Statistically significant differences across groups.

related covariates including depression. These findings are consistent with the existing literature supporting an association between chronic pain and impaired executive function (see meta-analysis by Berryman et al, $2014^{11}$ ), with greater levels of pain usually associated with greater levels of executive impairment. The association between pain and cognition in PD was also reported by Okada and colleagues (2016), who found decreased amplitudes of pain-related somatosensory evoked potentials and impaired attention and memory in individuals with PD. ${ }^{35}$ Some prior research, however, did not find an association between executive functioning and pain. Engels and colleagues (2016) reported that mood, and not any measured cognitive construct, was a significant predictor of pain in individuals with PD. However, this study did not assess pain interference directly, ${ }^{36}$ and this is consistent with the similar pain severity across the clusters in the present study.

Associations between executive function and pain interference are theoretically consistent with neurophysiology. Prefrontal brain regions are crucial for both executive function and pain inhibition and modulation, and the prefrontal cortex changes in persons with PD. ${ }^{14,37,38}$ Individuals with PD show more activity in cingulate and precuneus regions during painful stimulation compared to age-matched nonPD peers, ${ }^{39,40}$ and individuals with PD and persistent pain have thinner prefrontal and frontal cortex than individuals with PD and no persistent pain. ${ }^{41}$ Alterations to frontal lobe integrity (outside of atrophy alone) may account for our observed differences in pain interference without significant 
differences in pain severity; there are known neuroanatomical differences in the processing of pain severity versus its perception of interference. ${ }^{14,42-44}$ Frontal regions are highly interconnected with subcortical regions involved in pain modulation $^{14}$ and emotional regulation for appropriately directing behavior.

Study limitations include the sample size, homogeneous sample from a diversity and geographic standpoint and reliance upon self-report measures of pain versus experimental measures of pain response. Additionally, all testing was conducted on-dopaminergic medication in order to achieve the best test performance. We encourage future research examining off-medication pain and cognitive profiles, as well as interactions between pain, cognition, and motor presentation. Strengths include the well-characterized cohort of individuals and considerations of two common cognitive complaints (reduced attention and memory) in relation to pain. Our study provides initial evidence for the association between frontal function (working memory, inhibition) cognitive symptoms in PD and the self-report of pain interference. Pain interference is a core component of health-related quality of life in assessing pain treatments, ${ }^{45}$ representing an important domain that should be targeted for psychosocial interventions in persons with PD. Future longitudinal studies are needed to decipher the directionality of these findings and the neurobiological substrates accounting for the high pain interference in individuals with PD and executive function. Such multifactorial approach may also lead to development of evidence-based pain treatments that are tailored to individuals' cognitive profiles.

\section{Acknowledgments}

The authors would like to acknowledge Dr Andrew Ahn for his initial ideas and early discussions of this work, Katie Rodriguez for her excellent work as a study coordinator, and colleagues within the Norman Fixel Institute for Neurological Diseases who help make work on PD possible at our institution. This work was supported in part by funding from NIH/ NINDS (R01NS082386 to Dr Catherine Price) and NIH/NIA (K01AG048259, R01AG059809, R01AG067757 to Dr Cruz-Almeida supporting her time).

\section{Disclosure}

All authors report no conflict of interest.

\section{References}

1. Aarsland D, Bronnick K, Williams-Gray C, et al. Mild cognitive impairment in Parkinson disease: a multicenter pooled analysis. Neurology. 2010;75:1062-1069.

2. Goldman JG, Weis H, Stebbins G, Bernard B, Goetz CG. Clinical differences among mild cognitive impairment subtypes in Parkinson's disease. Mov Disord. 2012;27:1129-1136.

3. Goldman JG, Aggarwal NT, Schroeder CD. Mild cognitive impairment: an update in Parkinson's disease and lessons learned from Alzheimer's disease. Neurodegener Dis Manag. 2015;5:425-443.

4. Tanner JJ, Mareci TH, Okun MS, Bowers D, Libon DJ, Price CC. Temporal lobe and frontal-subcortical dissociations in non-demented Parkinson's disease with verbal memory impairment. PLoS One. 2015;10:e133792.

5. Price CC, Tanner J, Nguyen PT, et al. Gray and white matter contributions to cognitive frontostriatal deficits in non-demented Parkinson's disease. PLoS One. 2016;11:e0147332.

6. Broen MP, Braaksma MM, Patijn J, Weber WE. Prevalence of pain in Parkinson's disease: a systematic review using the modified QUADAS tool. Mov Disord. 2012;27:480-484.

7. Beiske AG, Loge JH, Ronningen A, Svensson E. Pain in Parkinson's disease: prevalence and characteristics. Pain. 2009;141:173-177.

8. Defazio G, Berardelli A, Fabbrini G, et al. Pain as a nonmotor symptom of Parkinson disease: evidence from a case-control study. Arch Neurol. 2008;65:1191-1194.

9. Negre-Pages L, Regragui W, Bouhassira D, Grandjean H, Rascol O. Chronic pain in Parkinson's disease: the cross-sectional French DoPaMiP survey. Mov Disord. 2008;23:1361-1369.

10. Young Blood MR, Ferro MM, Munhoz RP, Teive HA, Camargo CH. (2016) Classification and characteristics of pain associated with Parkinson's disease. Parkinsons Dis. 2016;6067132.

11. Berryman C, Stanton TR, Bowering KJ. Do people with chronic pain have impaired executive function? A meta-analytical review. Clin Psychol Rev. 2014;34:563-579.

12. Oosterman JM, Dijkerman HC, Kessels RP, Scherder EJ. A unique association between cognitive inhibition and pain sensitivity in healthy participants. Eur.J.Pain. 2010;14:1046-1050.

13. Bjekic J, Zivanovic M, Puric D, Oosterman JM, Filipovic SR. Pain and executive functions: a unique relationship between Stroop task and experimentally induced pain. Psychol Res. 2017;1:15.

14. Tracey I, Mantyh PW. The cerebral signature for pain perception and its modulation. Neuron. 2007;55:377-391.

15. Alexander GE, DeLong MR, Strick PL. Parallel organization of functionally segregated circuits linking basal ganglia and cortex. Annu Rev Neurosci. 1986;9:357-381.

16. Crowley SJ, Banan G, Amin M. Statistically defined Parkinson's Disease executive and memory cognitive phenotypes: demographic, behavioral, and structural neuroimaging comparisons. J Parkinson's Dis. 2015;2:1254

17. Crowley SJ, Hizel L, Tanner JJ, Cruz-Almeida Y, Price CC. In International Neuropsychological Society. Washington, DC; 2018.

18. Hughes AJ, Ben-Shlomo Y, Daniel SE, Lees AJ. What features improve the accuracy of clinical diagnosis in Parkinson's disease: a clinicopathologic study. Neurology. 1992;42:1142-1146.

19. Hoehn MM, Yahr MD. Parkinsonism: onset, progression and mortality. Neurology. 1967;17:427-442.

20. Reijnders JS, Ehrt U, Weber WE, Aarsland D, Leentjens AF. A systematic review of prevalence studies of depression in Parkinson's disease. Mov Disord. 2008;23:183-189.

21. den Brok MG, van Dalen JW, van Gool WA. Apathy in Parkinson's disease: a systematic review and meta-analysis. Mov Disord. 2015;30:759-769.

22. Jankovic J, McDermott M, Carter J, et al. Variable expression of Parkinson's disease: a base-line analysis of the DATATOP cohort. The Parkinson Study Group. Neurology. 1990;40:1529-1534. 
23. Cleeland CS. Measurement of pain by subjective report. In: $\mathrm{Cr} \mathrm{C}$, Jd L, editors. Advances in Pain Research and Therapy Vol. 12: Issues in Pain Management. New York: Raven Press; 1989:391-403.

24. Charlson ME, Pompei P, Ales KL, MacKenzie CR. A new method of classifying prognostic comorbidity in longitudinal studies: development and validation. J Chronic Dis. 1987;40:373-383.

25. Sheikh JA, Yesavage JA. Geriatric Depression Scale (GDS): recen evidence and development of a shorter version. Clin Gerontol. 1986.

26. Tomlinson CL, Stowe R, Patel S, Rick C, Gray R. Clarke CE Systematic review of levodopa dose equivalency reporting in Parkinson's disease. Mov Disord. 2017;25:2649-2653.

27. Carnahan RM, Lund BC, Perry PJ, Pollock BG, Culp KR. The Anticholinergic Drug Scale as a measure of drug-related anticholinergic burden: associations with serum anticholinergic activity. J Clin Pharmacol. 2006;46:1481-1486.

28. Wechsler D. WAIS-III Administration and Scoring Manual (3rd Ed). San Antonio, TX: The Psychological Corporation; 1997.

29. Jensen AR, Rohwer WD. The Stroop color-word test: a review. Acta Psychol (Amst). 1966;25:36-93.

30. Spreen O, Strauss E. A Compendium of Neuropsychological Tests: Administration, Norms, and Commentary. New York: Oxford University Press; 1991.

31. Benedict RHB, Schretlen D, Groninger L, Brandt J. Hopkins Verbal Learning Test - revised: normative Data and Analysis of Inter-Form and Test-Retest Reliability. Clin Neuropsychol. 1998;12:43-55.

32. Wechsler D. WAIS-III: Wechsler Adult Intelligence Scale. Psychological Corporation; 1997.

33. Zis P, Daskalaki A, Bountouni I, Sykioti P, Varrassi G, Paladini A. Depression and chronic pain in the elderly: links and management challenges. Clin Interv Aging. 2017;12:709-720.

34. Rock PL, Roiser JP, Riedel WJ, Blackwell AD. Cognitive impairment in depression: a systematic review and meta-analysis. Psychol Med. 2014;44:2029-2040.
35. Okada A, Nakamura T, Suzuki J, et al. Impaired pain processing correlates with cognitive impairment in Parkinson's disease. Intern Med. 2016;55:3113-3118.

36. Engels G, Weeda WD, Vlaar AM, Weinstein HC, Scherder EJ. Clinical pain and neuropsychological functioning in Parkinson's disease: are they related? Parkinsons Dis. 2016;(2016):8675930.

37. Hanganu A, Bedetti C, Degroot C, et al. Mild cognitive impairment is linked with faster rate of cortical thinning in patients with Parkinson's disease longitudinally. Brain. 2014;137:1120-1129.

38. Tinaz S, Courtney MG, Stern CE. Focal cortical and subcortical atrophy in early Parkinson's disease. Mov Disord. 2011;26:436-441.

39. Aschermann Z, Nagy F, Perlaki G, et al. 'Wind-up' in Parkinson's disease: a functional magnetic resonance imaging study. Eur J Pain. 2015;19:1288-1297.

40. Forkmann K, Grashorn W, Schmidt K, Frundt O, Buhmann C, Bingel U. Altered neural responses to heat pain in drug-naive patients with Parkinson disease. Pain. 2017;158:1408-1416.

41. Polli A, Weis L, Biundo R, et al. Anatomical and functional correlates of persistent pain in Parkinson's disease. Mov Disord. 2016;31:1854-1864

42. Levesque J, Eugene F, Joanette Y, et al. Neural circuitry underlying voluntary suppression of sadness. Biol Psychiatry. 2003;53:502-510.

43. Ohira H, Nomura M, Ichikawa N, et al. Association of neural and physiological responses during voluntary emotion suppression. Neuroimage. 2006;29:721-733.

44. Apkarian AV, Bushnell MC, Treede RD, Zubieta JK. Human brain mechanisms of pain perception and regulation in health and disease. Eur J Pain. 2005;9:463-484.

45. Dworkin RH, Turk DC, Wyrwich KW. Interpreting the clinical importance of treatment outcomes in chronic pain clinical trials: IMMPACT recommendations. J Pain. 2008;9:105-121.
Journal of Pain Research

\section{Publish your work in this journal}

The Journal of Pain Research is an international, peer reviewed, open access, online journal that welcomes laboratory and clinical findings in the fields of pain research and the prevention and management of pain. Original research, reviews, symposium reports, hypothesis formation and commentaries are all considered for publication. The manuscript management system is completely online and includes a very quick and fair peer-review system, which is all easy to use. Visit http:// www.dovepress.com/testimonials.php to read real quotes from published authors. 